\title{
Study of water Cherenkov detector to determine air shower arrival directions with accuracy
}

\author{
A. Shiomi, ${ }^{a, *}$ H. Nakada, ${ }^{b}$ Y. Katayose,${ }^{b}$ M. Ohnishi, ${ }^{c}$ T. K. Sako ${ }^{c}$ and K. Hibino ${ }^{d}$ \\ ${ }^{a}$ Nihon University, College of Industrial Technology, \\ 275-8575, Narashino, Japan \\ ${ }^{b}$ Yokohama National University, Faculty of Engineering, \\ 240-8501, Yokohama, Japan \\ ${ }^{c}$ University of Tokyo, Institute for Cosmic Ray Research, \\ 277-8582, Kashiwa, Japan \\ ${ }^{d}$ Kanagawa University, Faculty of Engineering, \\ 221-8686, Yokohama, Japan \\ E-mail: shiomi.atsushi@nihon-u.ac.jp
}

Experiments with high angular resolution that can detect gamma rays in the $100 \mathrm{TeV}$ region will help study acceleration mechanisms of gamma-ray sources with morphological characteristics. In recent years, three groups using extensive air-shower (AS) arrays have reported detecting gamma rays in the $100 \mathrm{TeV}$ region from celestial bodies in the galaxy. In extensive air shower experiments, an arrival direction of a cosmic ray is determined by estimating a shape of a front surface of an air shower based on the density distribution and detection time of secondary particles. The density of secondary gamma rays of an air shower is several times that of secondary electrons and positrons in an air shower and the difference increases as the distance from the shower axis increases. Therefore, increasing the sensitivity to detect secondary gamma rays plays an important role in determining the arrival direction of the shower. Monte Carlo simulation was used to investigate the effect of secondary gamma rays on the time determination accuracy of the air shower front. When an AS array capable of measuring secondary gamma rays with $100 \%$ sensitivity was installed at an altitude of $4,740 \mathrm{~m}$, the angular resolution for a $500 \mathrm{TeV}$ shower was improved by about $40 \%$ compared to an array that could observe only secondary electrons and positrons. The optimum shape of the water Cherenkov detector (WCD), which is highly sensitive to gamma rays, was investigated by Monte Carlo simulation. When a water tank with a radius of $4.5 \mathrm{~m}$ and PMTs with a diameter of 8 inches was used, it was found that about 19 PMTs (area ratio of approximately 0.01 ) were suitable for detection efficiency and timing response at a water depth of $1.6 \mathrm{~m}$.

$37^{\text {th }}$ International Cosmic Ray Conference (ICRC 2021)

July 12th - 23rd, 2021

Online - Berlin, Germany

\footnotetext{
${ }^{*}$ Presenter
} 


\section{Introduction}

Cosmic rays up to the knee region are mainly considered to be accelerated by supernova remnants in our galaxy. The energy of gamma rays from the $\pi^{0}$ decay produced by the interaction of cosmic rays in the knee region with the interstellar medium near the acceleration site is expected to be in the $100 \mathrm{TeV}$ region. In recent years, Tibet AS $\gamma$, HAWC and LHAASO groups using extensive air-shower (AS) arrays have reported detecting gamma rays in the $100 \mathrm{TeV}$ region from celestial bodies in the galaxy. The Tibet AS $\gamma$ experiment detected gamma rays above $100 \mathrm{TeV}$ from the supernova remnant G106.3 +2.7 [1]. The gamma-ray emission region coincides with the position of the molecular cloud $0.44^{\circ}$ away from the pulsar PSRJ2229 + 6114 and supports the hadron origin due to the $\pi^{0}$ decay caused by relativistic protons. As can be seen from this result, measuring the gamma-ray emission region with high angular resolution, especially for celestial bodies with morphological characteristics, greatly contributes to understanding the cosmic ray acceleration mechanism. In extensive air shower experiments, an arrival direction of a cosmic ray is determined by estimating a shape of a front surface of an air shower based on the density distribution and detection time of secondary particles. The density of secondary gamma rays of an air shower is several times that of secondary electrons and positrons in an air shower, and the difference increases as the distance from the shower axis increases. Therefore, increasing the sensitivity to detect secondary gamma rays plays an important role in determining the arrival direction of the shower [2], [3]. We report the results of investigating the effect of secondary gamma rays on the time determination accuracy of an air shower front using Monte Carlo simulation. We also report the results of examining different detector structures to improve the time resolution using a WCD, which has high detection efficiency for secondary gamma rays.

\section{Simulation}

Using Monte Carlo simulation, we investigated an air shower observation method for measuring cosmic gamma rays in the energy region of several tens of $\mathrm{TeV}$ or more with high angular resolution. Air showers are generated with the CORSIKA Ver.7.6400 code and Geant4 10.04.p02 simulates detector responses. Table 1 shows the simulation settings used [4], [5].

Table 1: Simulation settings

\begin{tabular}{lcc}
\hline Section & 2.1 and 2.2 & 2.3 \\
\hline \hline Primary particles & $500 \mathrm{TeV}$ gamma rays & $10 \mathrm{TeV}$ gamma rays \\
\hline Air shower core & $\begin{array}{c}\text { Randomly within } 10 \mathrm{~m} \\
\text { from the array center }\end{array}$ & 1000 \\
\hline Total number of events & $1000 \times 100$ (over sampling) & $0.773 \mathrm{MeV}$ \\
\hline Minmum energy & $1 \mathrm{MeV}$ & $0^{\circ}$ \\
\hline Zenith angle & $0^{\circ}$ & Fastest (within 1 ns) secondary particles \\
\hline Other selection & & $30 \mathrm{~m}$ away from the shower core \\
\hline
\end{tabular}




\subsection{Characteristics of the electromagnetic component of the induced air shower front}

An ideal electromagnetic shower is composed of a large number of particles with a lateral spread of particle density represented by the NKG function [6]. It is known that at a certain distance from the shower axis, the energy of gamma rays exceeds the energy of electrons, and the difference increases as the distance from the shower axis increases. As a result, the ratio of the number of secondary gamma rays to the number of secondary electrons increases as the distance from the shower axis increases.

In experiments using surface detector arrays, the time of shower front particles incident on each detector is used to estimate the arrival direction of cosmic rays. Therefore, the lateral spread of the particle number density affects the accuracy of the determined direction. It is expected that it will be especially important to measure secondary gamma rays far away from the shower axis. To investigate the detailed characteristics of the lateral density and arrival time distributions of shower particles, the characteristics of secondary particles with a minimum energy of $1 \mathrm{MeV}$ at an altitude of 4,740 m generated by vertically incident $500 \mathrm{TeV}$ gamma rays were investigated.

Regarding the lateral density distribution, the numbers of secondary gamma rays and secondary electrons and positrons per square meter below $100 \mathrm{MeV}$ estimated near $60 \mathrm{~m}$ and $120 \mathrm{~m}$ from the shower core are approximately 90,20, and approximately 20,3, respectively. The numbers of secondary gamma rays and secondary electrons and positrons per square meter above $100 \mathrm{MeV}$, which is larger than the critical energy of the electron, estimated near $60 \mathrm{~m}$ and $120 \mathrm{~m}$ from the shower core, are approximately 5, 2, and approximately $0.5,0.1$, respectively. If the effective area of the detector for gamma rays is 10 square meters or more, it is possible to detect secondary particles produced by $500 \mathrm{TeV}$ gamma rays even near $120 \mathrm{~m}$ from the shower core.

Regarding arrival time distribution, the distribution of gamma rays became slightly sharper near the center of the shower, and the secondary gamma rays tended to be concentrated in the front. When the distance from the shower core was around $120 \mathrm{~m}$, the difference between the distribution of secondary gamma rays and secondary electrons was small. However, the number of secondary gamma rays is several times that of secondary electrons, so the detector is more likely to detect early-arriving secondary gamma rays.

\subsection{Verification of the effect of secondary gamma rays on direction determination}

In experiments with surface detector arrays, the position of the air shower core is mainly determined from the density distribution of secondary particles, and the arrival direction of the shower is determined from the core position and relative timing data between multiple detectors. The data in Table 1 were used to investigate the effects of secondary gamma rays and electrons on the accuracy of the shower arrival direction. To investigate the effect of secondary gamma rays in determining the arrival direction of an air shower, the performance of two types of AS arrays consisting of virtual scintillation detectors with different characteristics was compared. One is an AS array with a virtual scintillation detector $\left(\operatorname{Det}_{\mathbf{A}}\right)$ that detects only electrons and positron. The other is an AS array with a virtual scintillation detector ( Det $_{\mathbf{B}}$ ) that converts gamma rays into electrons. Figure 1 and Figure 2 show the structure of the scintillation detector and the schematic view of the AS array, respectively. Primary particles randomly fall within a radius of $10 \mathrm{~m}$ from 
the center of each array, and the behavior of each detector, when exposed to electrons and gamma rays above $1 \mathrm{MeV}$, was calculated using Geant 4 .

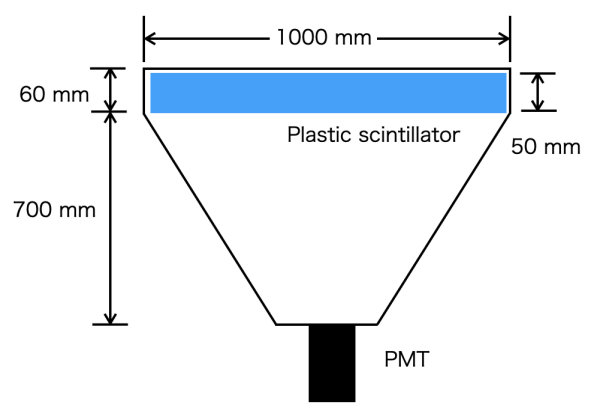

Figure 1: The basic structure of two virtual detectors ( Det $_{\mathbf{A}}$ and Det $\mathbf{B}_{\mathbf{B}}$ ) consisting of a plastic scintillator with an area of $1 \mathrm{~m}^{2}$ and a thickness of $5 \mathrm{~cm}$ and a photomultiplier tube.

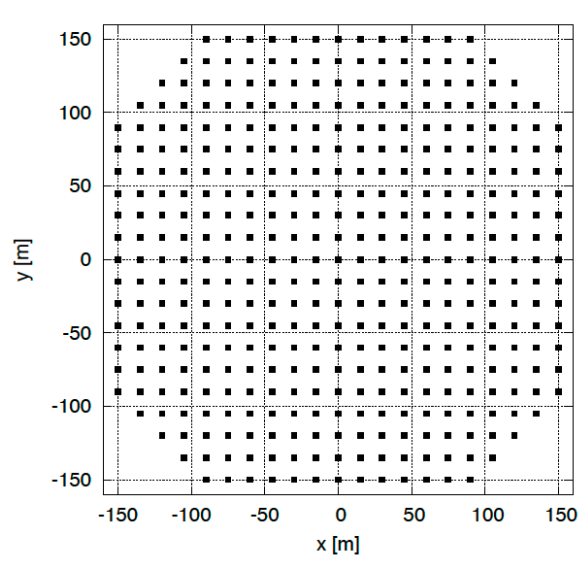

Figure 2: Schematic view of the AS arrays. The black squares represent the locations of 401 virtual plastic scintillation detectors installed at $15 \mathrm{~m}$ intervals.

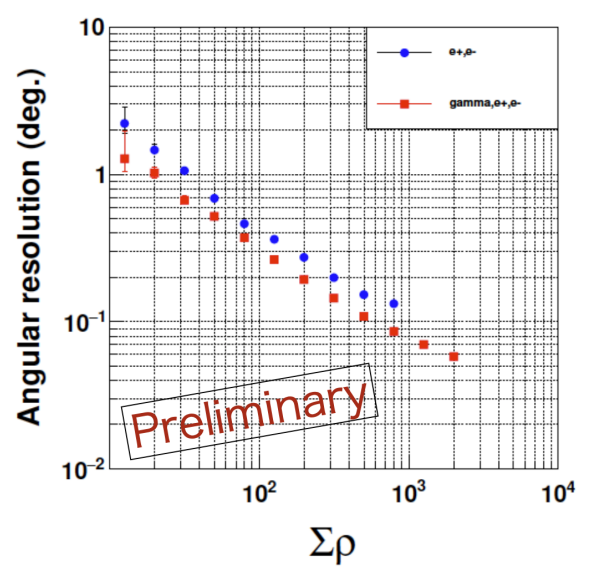

Figure 3: Dependence of angular resolution on the number of detected particles when a primary gamma ray of $500 \mathrm{TeV}$ is incident from the zenith. The horizontal and vertical axis represents the number of detected particles and angular resolution, respectively. When any four scintillation detectors detect more than 0.8 particles, the data is employed. The wide horizontal axis range $(\Sigma \rho)$ is achieved by removing the secondary particles produced by each primary particle by a fixed percentage from 0 to $99 \%$.

The dependence of the angular resolution of the air shower on the total number of measured particles $(\Sigma \rho)$ when a primary gamma ray of $500 \mathrm{TeV}$ is incident from the zenith. When any four scintillation detectors detect more than 0.8 particles, the data is employed. The wide range of the horizontal axis $(\Sigma \rho)$ is achieved by removing the secondary particles produced by each primary particles by a fixed percentage from 0 to $99 \%$ (equivalent to the size of the detector between $1 \mathrm{~m}^{2}$ to $0.01 \mathrm{~m}^{2}$ ). As is known, the angular resolution increases in inverse proportion to the square root of 
the number of particles measured. It was also found that secondary gamma rays contribute better angular resolution than electrons when the total number of particles measured is the same. Det $\mathbf{B}$ showed an improvement in angular resolution of about $40 \%$ over a $500 \mathrm{TeV}$ shower compared to Det $_{\mathbf{A}}$. The maximum value of $\Sigma \rho$ in the array of $\operatorname{Det}_{\mathbf{B}}$ that detects secondary gamma rays exceeds 1000 , and the angular resolution has improved to approximately $0.06^{\circ}$. Since the maximum value of $\Sigma \rho$ in the array of $\operatorname{Det}_{\mathbf{A}}$, which measures only secondary electrons and positrons, is approximately 800 , it can be seen that the angular resolution is improved by approximately 2 times or more.

\subsection{Optimal water Cherenkov detector design for measuring air shower fronts}

The WCD is highly sensitive to gamma rays. The detection efficiency and timing response of a WCD with 8-inch diameter PMTs installed at the bottom of a water tank with a radius of $4.5 \mathrm{~m}$ were investigated by Monte Carlo simulation. The reflectance on the side surface of the tank was set to $0 \%$. Figure 4 and Figure 5 show an example of the schematic view of the WCD and the arrangement of the PMTs, respectively.

Secondary gamma rays and electrons created with the Table 1 setting were uniformly incident on the upper surface of the WCD with different water depths and the number of PMTs installed. If any PMT detects three or more photoelectrons, it is judged to be "detected". In the case of a water depth of $1.6 \mathrm{~m}$, which is considered to be cost-effective, and a learning area ratio of 0.0095 using 19 PMTs, detection efficiencies of about 0.38 and 0.76 were obtained for vertically incident gamma rays and electrons, respectively. And the detection time error of secondary gamma rays is approximately $2.2 \mathrm{~ns}$.

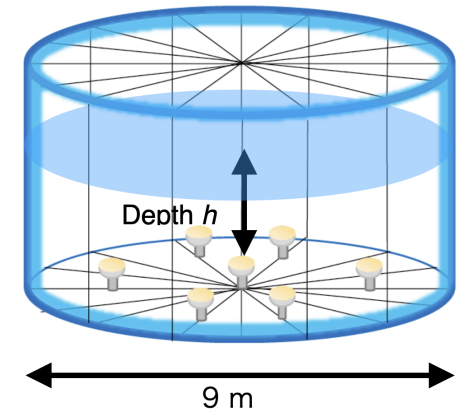

Figure 4: Schematic view of a WCD. WCD is made by $3.0 \mathrm{~mm}$-thick stainless steel water tank of $9.0 \mathrm{~m}$ diameter. Upward-facing 8 " PMTs are installed on the floor. The light reflectance of the inner wall of the tank is $0 \%$.

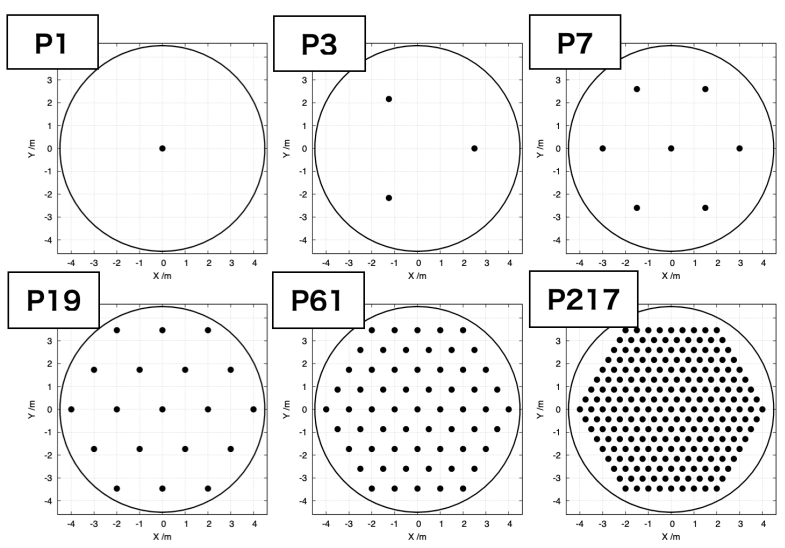

Figure 5: Arrangement of PMTs used in the simulation. PMTs were placed at equal intervals in a circle with a radius of $4.5 \mathrm{~m}$. The performance of the WCD was evaluated in six cases. When the number of PMTs is i, a label of " $\mathrm{Pi}$ ” is applied.

\section{Conclusion}

Since the secondary gamma rays are more widely distributed than the secondary electrons, it was confirmed that the angular resolution can be improved by efficiently detecting the gamma rays 
and widely deploying a detector for increasing the detected number of particles. By operating a well-placed WCD array in combination with an AS array consisting of scintillation detectors that can measure each air showers' core position and energy, it will be able to observe primary gamma rays in the energy range of 10 to $1000 \mathrm{TeV}$ with high angular resolution.

\section{Acknowledgments}

This work was supported by JSPS KAKENHI Grant Number JP15K05108.

\section{References}

[1] M. Amenomori, et al., Potential PeVatron supernova remnant G106.3+2.7 seen in the highestenergy gamma rays, Nature Astronomy (2021). https://DOI.org/10.1038/s41550-020-01294-9

[2] J. Poirier, S. Mikochi, Improving the angular resolution of existing air shower arrays by adding a thin layer of lead, NIM A, 257, 473 (1987)

[3] M. Amenomori, et al., Development and performance test of a prototype air shower array for search for gamma ray point sources in the very high energy region, NIM A, 288, 619 (1990)

[4] D. Heck et al., CORSIKA: A Monte Carlo ode to Simulate Extensive Air Showers, Report FZKA, 6019, Forschungszentrum Karlsruhe (1998)

[5] S. Agostinelli, et al. GEANT4 - a simulation toolkit, Nucl. Instrum. Methods Phys. Res. A, $506250(2003)$

[6] J. Nishimura and K. Kamata, The Lateral and the Angular Structure Functions of Electron Shower, Progr. Theor. Phys. Suppl., 6,93 (1958) 